Ann. Génét. Sél. anim., r975, 7 (3), 303-3ro.

\title{
ÉVOLUTION DE LA FERTILITÉ ET DE LA VIABILITÉ DE POPULATIONS EXPÉRIMENTALES DE DROSOPHILA MELANOGASTER DÉRIVÉES D'UN SEUL COUPLE
}

\author{
C. BIÉMONT \\ Laboratoire de Biométrie, \\ Département de Biologie générale et appliquée, \\ Université Claude-Bernard, Lyon I, \\ 69621 Villeurbanne (France)

\section{RÉSUMÉ}

Des sous-populations dérivées d'un couple atteignent des valeurs d'équilibre de la fertilité et de la viabilité identiques à celles des populations sauvages d'origine de ces couples; seule l'évolution vers cet équilibre dépend des caractéristiques des femelles fondatrices. Ces femelles possèdent donc toutes les " potentialités " nécessaires pour l'établissement d'une nouvelle population. Ces résultats sont en accord avec les prévisions de la théorie chromosomique de la consanguinité ; ils soulèvent le problème des populations d'effectif limité et de la colonisation de nouveaux territoires par un faible nombre d'individus. Nous concluons que la pression de consanguinité dans les populations de faible effectif, l'influence de l'environnement si l'effectif devient grand, doivent être responsables des divergences observées entre de telles populations dérivées d'un couple. Au niveau d'équilibre, le premier type de population est peu ou pas sensible à la consanguinité, tandis que le deuxième type est sensible.

\section{INTRODUCTION}

Le problème des populations d'effectif limité ou dérivées d'un nombre réduit de couples extrait d'une vaste population (à la limite un seul couple peut donner naissance à une population) a fait l'objet d'un certain nombre d'études théoriques et expérimentales (Dobzhansky et Pavlovsky, I957; Solima-Simmons, I966 ; Parsons et Hosgood, r967 ; Crow et Kimura, I970 ; Hosgood et Parsons, I97I). Les études de laboratoire montrent que l'hétérogénéité, au niveau des caractères biométriques considérés, est plus grande dans les populations dérivées d'un petit 
nombre de couples que dans celles dérivées d'un nombre de fondateurs important. L'effet de fondateur (MAYR, I963) est considéré comme responsable des modifications des caractéristiques moyennes de ces sous-populations dont le maintien (qui dépend d'une pression de consanguinité théoriquement élevée) est rendu possible par la sélection favorisant les individus les plus hétérozygotes (FISCHER, I949) ; cette hétérozygotie serait d'autre part favorisée par une vitesse élevée de croissance de la population permettant selon NEY et al. (I975) l'incorporation de nouvelles mutations.

Dans ces expériences, les populations sont généralement analysées après un grand nombre de générations de sorte qu'une sélection involontaire jointe aux fluctuations du milieu extérieur peut entraîner l'apparition d'une diversité entre ces populations. D'autre part, les caractéristiques étudiées (inversions et polymorphisme chromosomiques, caractères biométriques morphologiques) sont très complexes et ne sont pas soumises à l'influence d'une balance génique efficace. Par contre on sait peu de chose sur le comportement au cours du temps de la fertilité et de la viabilité de ces populations, ces deux caractères étant pourtant fondamentaux pour leur survie. Une telle recherche est d'autant plus justifiée que l'analyse mathématique de la théorie chromosomique de la consanguinité (impliquant un contrôle du développement embryonnaire par un système d'interactions chromosomiques : BiÉmonT et al., r974; BIÉmon'r, I974 $b$ ) donne une approche possible de cet effet de fondateur, dans le cas des caractères liés à la fitness. BoUfFETTTE et BoUFFETTE (I975) ont en effet montré que lorsqu'une population naturelle panmictique est en état d'équilibre (au niveau de la composition en sites actifs et inactifs), la fréquence des individus présentant une structure donnée est constante et cet équilibre est indépendant de la structure des fondateurs initiaux. Si les paramètres contrôlant cet équilibre sont caractéristiques de l'espèce et non de la femelle, alors une population dérivée d'un couple doit, au bout de $n$ générations, atteindre le même équilibre que la population initiale ; seule l'évolution vers cet équilibre dépend de la structure génétique de la femelle fondatrice. Pour tester ces hypothèses, trois populations ont été établies à partir de trois femelles prélevées dans trois souches sauvages différentes et connues de Drosophila melanogaster. Dans cet article, nous suivons l'évolution de la fertilité et de la viabilité de ces populations au cours des générations.

\section{MATÉRIEL ET MÉTHODE}

Nous disposons de trois souches sauvages de Drosophila melanogaster prélevées récemment dans la nature et dont les caractéristiques physiologiques de fertilité et viabilité sont connues (Bı́́mont, I972, I974a). Ces souches appelées Varces, Tours et Saint-Just selon le lieu de leur capture sont maintenues par élevage en masse, à l'obscurité, à une température de $25^{\circ} \mathrm{C}$, dans des erlens de $250 \mathrm{ml}$ contenant environ $50 \mathrm{ml} \mathrm{du}$ milieu nutritif axénique de David (I959).

Pour chaque souche, une femelle fécondée et fertile a été isolée au hasard et nous avons déterminé le taux d'éclosion de ses œufs (fertilité) ainsi que le taux d'émergence des adultes obtenus à partir des œufs éclos (viabilité). Les descendants adultes de chaque femelle ont ensuite été laissés libres de s'accoupler entre eux, ainsi à la première génération les individus se croisent tous entre frère et sœur. Chaque nouvelle génération est obtenue par repiquage dans un erlen contenant du milieu frais, d'un lot d'environ 500 œufs de la génération précédente ; il n'y a donc pas de chevauchement entre les générations.

La fertilité et la viabilité d'une trentaine de couples fertiles ont été testées à chaque génération. Ces couples, prélevés dans la sous-population, après anesthésie à l'éther, sont isolés dans des tubes $\left(\mathrm{r}_{5} \times 75 \mathrm{~mm}\right)$ contenant du milieu nutritif de manière à s'assurer de leur fertilité. Les 
couples fertiles sont ensuites transvasés dans des boîtes de plastique $(50 \times 30 \times 15 \mathrm{~mm})$ contenant une mince couche de milieu ; ceci permet un dénombrement facile des cufs pondus pendant Io $h$ (une femelle de 4 à 5 jours pond ainsi de 30 à 40 œufs par boîte) ainsi que des adultes obtenus en fin de développement.

\section{RÉSULTATS}

Les deux caractères de fertilité et viabilité ont été choisis en fonction de leur grande sensibilité à la consanguinité et pour le fait qu'ils répondent indépendamment pour un même individu et selon le sens du croisement consanguin considéré (BIÉMONT, 1972).

\section{TABLEAU I}

Effets du système de croisement frère-saur sur la fertilité et la viabilité des populations sauvages Tours et Saint-Just et des sous-populations Tours et Saint Just dérivées d'un couple, testées à l'équilibre

Fertilité : nombre d'œufs éclos $\times$ roo/nombre d'œufs étudié

Viabilité : nombre d'adultes $\times$ Ioo/nombre d'œufs éclos

L'analyse statistique entre témoins et consanguins a été réalisée par le test $\varepsilon$ de comparaison de fréquences

$\mid \begin{gathered}\begin{array}{c}\text { Nombre d'œufs } \\ \text { étudié }\end{array} \\ \text { Fiabilité }\end{gathered}$

Souches sauvages $\left({ }^{1}\right)$

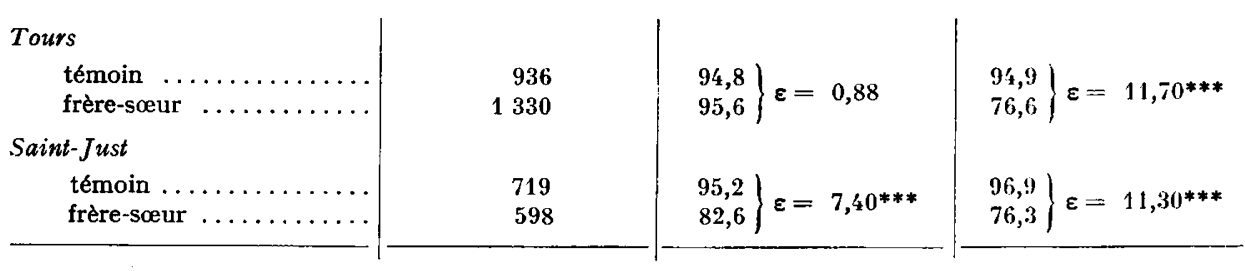

Sous-populations dérivées d'un seul couple

Sous-population Tours

témoin

frère-søur $\ldots \ldots \ldots \ldots \ldots \ldots$

témoin

frère-sœur $\ldots \ldots \ldots \ldots \ldots$

Sous-population Saint-Just

témoin $\ldots \ldots \ldots \ldots \ldots$

frère-sœur $\ldots \ldots \ldots \ldots \ldots$

témoin $\ldots \ldots \ldots \ldots \ldots \ldots$

frère-sœur

\begin{tabular}{|c|c|}
\hline $\begin{array}{l}93,5 \\
9^{\prime}, 9\end{array}$ & $\varepsilon=1,42$ \\
\hline $\begin{array}{l}96,7 \\
96,1\end{array}$ & $\varepsilon=0,70$ \\
\hline $\begin{array}{l}93,6 \\
87,9\end{array}$ & $\varepsilon=4,52^{* * *}$ \\
\hline & $\varepsilon=4,71 * * *$ \\
\hline
\end{tabular}$$
\left.\begin{array}{l}
94,6 \\
79,5
\end{array}\right\} \varepsilon=9,87 * * *
$$$$
\left.\begin{array}{l}
95,9 \\
77,6
\end{array}\right\} \varepsilon=12,09^{* * *}
$$$$
71,9\} \varepsilon=13,85^{* * *}
$$$$
\left.\begin{array}{l}
93, t \\
71,9
\end{array}\right\} \varepsilon=19,95 * * *
$$

*** Différence significative à $\mathrm{P}<0,001$.

(1) D'après Bí́mont (1974 $a$ ). 

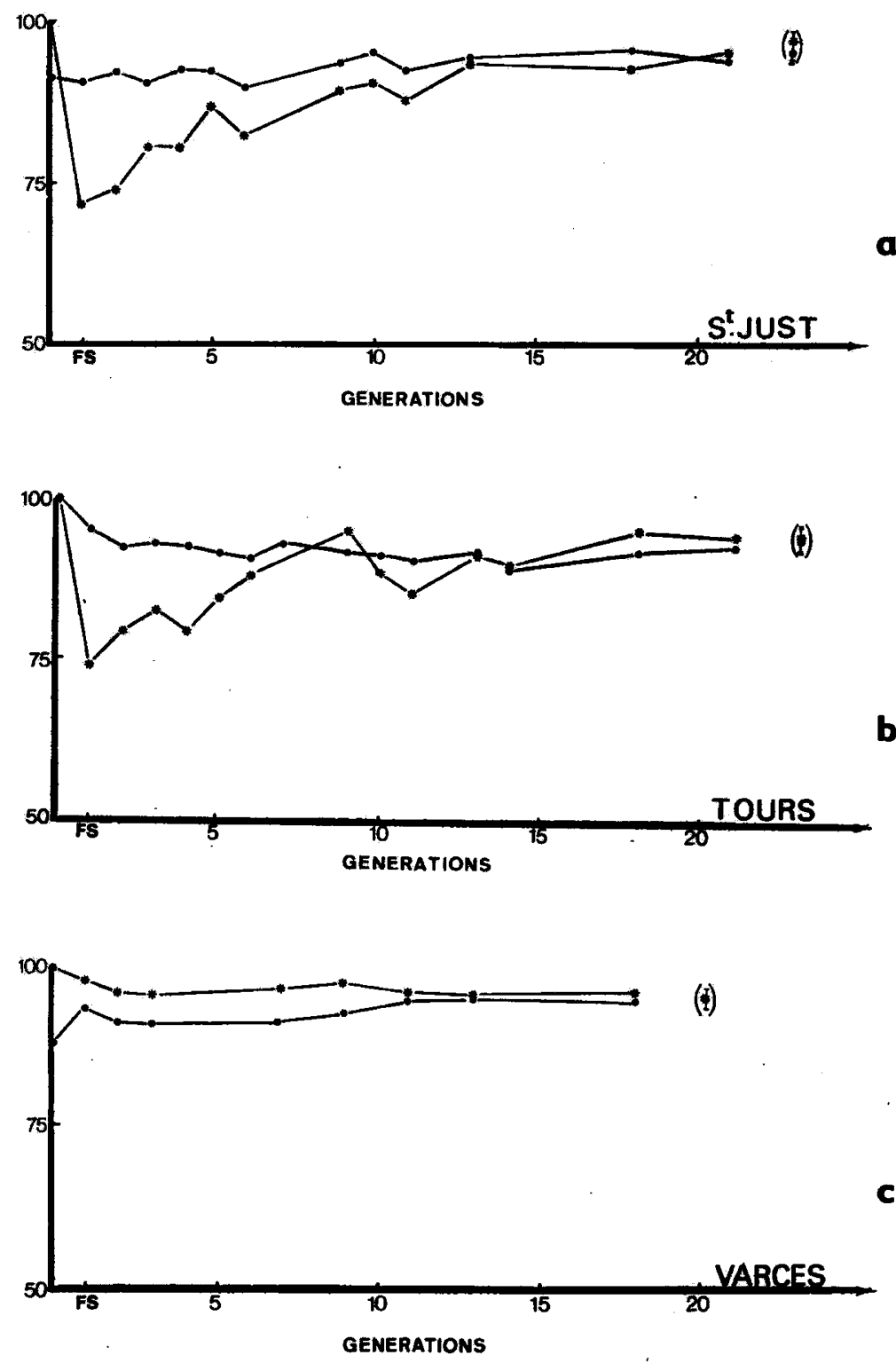

FIG. I. - Evolution au cours des générations de la fertilité et de la viabilite des trois sous-populations Saint-Just, Tours et Varces

- Fertilité : nombre d'œufs éclos $\times$ roo/nombre d'œufs étudié

- Viabilité : nombre d'adultes $\times$ roo/nombre d'cufs éclos

(•) et (*): fertilité et viabilité des populations sauvages initiales d'où ont été extraites les femelles 


\section{Évolution de la fertilité et de la viabilité au cours des générations}

La figure I représente l'évolution des taux d'éclosion des œufs (fertilité) et d'émergence des adultes (viabilité) pour les trois populations analysées. Les premières valeurs correspondent à la fertilité et à la viabilité de la femelle fondatrice ; elles se distinguent généralement assez nettement des valeurs moyennes des populations sauvages d'origine des femelles (tabl. I). Les deux femelles provenant des souches Varces et Saint-Just ont une fertilité assez faible, respectivement de 88 p. roo et $92 \mathrm{p}$. Ioo. Cette fertilité augmente dès la première génération pour Varces alors que tous les individus sont croisés entre frère et sœur; pendant les générations suivantes, elle se maintient toujours supérieure à celle de la femelle fondatrice et atteint une valeur d'équilibre à partir de la II $^{\mathbf{e}}$ génération; cette fertilité d'équilibre n'est pas statistiquement différente de la fertilité moyenne de la souche sauvage initiale (les valeurs observées au niveau du plateau d'équilibre appartiennent à l'intervalle de confiance de la fertilité moyenne de la population initiale (fig. I). La fertilité de la sous-population Saint-Just augmente légèrement au cours des générations et atteint une valeur d'équilibre correspondant à la valeur de la population sauvage vers la $I 3^{\mathrm{e}}$ génération. La femelle provenant de la souche Tours a une fertilité très élevée de Ioo $\mathrm{p}$. Ioo ; celle-ci diminue graduellement au cours des générations pour atteindre la valeur de la population initiale vers la $3^{\mathbf{e}}$ génération.

L'évolution de la viabilité est beaucoup plus caractéristique. Les trois femelles fondatrices ont un taux d'émergence des adultes de roo p. Ioo (fig. I) nettement supérieur aux taux moyens des souches sauvages (95 p. roo). Dès la première génération frère-sœur, la viabilité des sous-populations diminue très fortement pour Saint-Just et Tours et plus faiblement pour Varces; tandis que pour cette dernière la diminution se manifeste jusqu'à la génération $F_{3}$ pour laquelle la viabilité d'équilibre est atteinte, pour les sous-populations Saint-Just et Tours la viabilité augmente dès la $F_{2}$ et cette augmentation se maintient jusqu'à l'obtention de la valeur d'équilibre, vers les $5_{5}{ }^{\text {e }}$ et $\mathrm{I} 8^{\mathrm{e}}$ générations.

\section{Sensibilité à la consanguinité}

D'après le modèle mathématique présenté précédemment (BOUFFETTE et BOUFFETTE, I975), les sous-populations doivent posséder à l'équilibre les mêmes caractéristiques que les populations initiales et ainsi être également sensibles à la consanguinité. Pour vérifier cette hypothèse, nous avons analysé la sensibilité des deux sous-populations Tours et Saint-Just à des croisements entre frères et sœurs. Les résultats obtenus (tabl. I) montrent que ces deux populations répondent à la consanguinité d'une manière semblable à celle des deux populations sauvages initiales. La fertilité de la sous-population Saint-Just est cependant moins diminuée par les croisements frère-sœur que celle de la population sauvage ; ceci peut s'expliquer en supposant l'action d'une sélection en faveur d'individus insensibles à la consanguinité.

La sous-population Tours est comme la population sauvage initiale, insensible à la consanguinité au niveau de la fertilité (BIÉMONT, I974a). Cette insensibilité se transmet ainsi de génération en génération. 


\section{DISCUSSION}

L'ensemble de ces résultats nous amène à conclure que quelle que soit la valeur du couple fondateur (au point de vue fertilité et viabilité), la sous-population qui en dérive tend à acquérir une valeur d'équilibre stable correspondant à la valeur de la population sauvage d'où le couple a été extrait, toutes conditions du milieu extérieur étant semblables par ailleurs. Un seul couple possède donc des caractéristiques génétiques " potentielles » suffisantes pour recréer une nouvelle population. La variabilité initiale que l'on observe entre les femelles d'une même population ou de populations distinctes viendrait alors d'un arrangement différent de ces potentialités à l'intérieur du génome des individus. Cet arrangement se trouverait perturbé dès la première génération de croisement entre les descendants de la femelle (cette génération résulte de croisements frère-sœur) puis tendrait, au cours des générations suivantes vers un équilibre correspondant à des valeurs optimales de la fertilité et de la viabilité ; ces deux caractères semblent donc dépendre d'un système "génique balancé " qui les maintient à une valeur optimale tant que le système de croisement n'est pas de type consanguin. En effet, les croisements consanguins répétés à chaque génération aboutissent à des lignées dont la valeur d'équilibre (1" " inbred minimum " de KING, I9I8) est généralement différente de la valeur des populations non consanguines témoins. A cet équilibre, les lignées inbred ne sont plus sensibles à un nouvel accroissement de leur " degré " de consanguinité tandis que nos populations expérimentales dérivées d'une femelle restent très sensibles. L'insensibilité à la consanguinité de la fertilité de la souche Tours (BrémonT, I974 a), que l'on retrouve au niveau de la sous-population Tours, distingue nettement cette souche des deux autres Varces et Saint-Just. Ce phénomène montre que ces souches sont bien géographiquement distinctes; les résultats obtenus sur les trois populations ne peuvent donc pas être considérés comme une réponse particulière d'une " même population ".

Ainsi, il apparaît nécessaire de faire une discrimination entre les populations dérivées d'un nombre réduit de fondateurs et dont 1'effectif reste limité et celles dont l'effectif peut devenir grand au cours des générations. Les premières subissent une pression de consanguinité constante au cours du temps, ce qui peut aboutir à des divergences entre les populations elles-mêmes et par rapport à des populations d'effectif plus grand, ces populations ainsi formées sont d'autre part peu sensibles à la consanguinité. Les secondes peuvent subir une dépression, dès les premières générations, due aux liens d'apparentement des individus puis, elles vont rapidement atteindre une valeur identique à la population sauvage ; à ce seuil, elles sont sensibles à la consanguinité. Les conditions de milieu doivent cependant jouer un rôle très important pour la détermination et la fixation des caractéristiques de ces deux types de populations.

Selon Dobzhansky (I970) : "The immediate and obvious result of a population being reduced to very small numbers of individuals is, in normally outbred species, close inbreeding and loss of vigor. The ultimate result may be extinction. " L'existence, cependant, dans la nature, de telles populations est alors expliquée par 1'intervention d'une sélection en faveur des individus les plus hétérozygotes au dépend des 
homozygotes moins aptes à s'adapter à des conditions fluctuantes du milieu (BoESIGER, I969 ; PRAKASH, I973). NEY et al. (I975) supposent d'autre part qu'une vitesse élevée de croissance de la population, en permettant l'incorporation de nouvelles mutations, favoriserait le maintien de 1'hétérozygotie. La rapidité de la stabilisation de nos sous-populations, la divergence d'évolution entre les deux caractères de fertilité et de viabilité, l'effet " favorable " des croisements frère-sœur pour la souche Varces (fig. I) sont difficilement explicables par la sélection et les mutations. Ces résultats sont compatibles avec l'hypothèse de l'existence d'une " balance génique " efficace conduisant à une valeur " moyenne " de la population; ils sont également en accord avec les prédictions de l'analyse mathématique de la théorie chromosomique de la consanguinité (BOUFFETTE, et BOUFFETTE, I975).

Cet ensemble de résultats pose les problèmes : $a$ ) de la colonisation de territoires nouveaux par un petit nombre d'individus provenant d'une population plus vaste, b) des isolats constituant une grande population et qui sont soumis à la consanguinité car dérivés d'un nombre réduit de fondateurs et d'effectif faible, c) de la sensibilité à la consanguinité et de la structure génétique de populations dont l'effectif fluctue avec les saisons entre un minimum et un maximum et dont un accroissement en taille (par exemple en automne pour Drosophila melanogaster), peut être obtenu à partir de quelques mouches, $d$ ) du nombre effectif de reproducteurs, souvent assez faible, qui assure le renouvellement des populations à chaque génération. Pour les caractères liés à la " fitness ", les (le) fondateurs ne joueraient pas le rôle aussi déterminant que la plupart des auteurs leur attribue généralement; la valeur de l'équilibre de la population obtenue étant indépendante de leur " structure initiale ", celle-ci ne contrôlant que l'évolution vers cet équilibre. Par contre, la pression de consanguinité si l'effectif reste limité, les conditions de milieu si l'effectif devient très grand, doivent être responsables des différences possibles observées entre les populations qui s'établissent ainsi.

Reçu pour publication en octobre 1975.

\section{SUMMARY}

FERTILITY AND VIABILITY OF EXPERIMENTAL, DROSOPHILA MELANOGASTER POPULATIONS STARTED FROM A SINGLE COUPLE

\footnotetext{
Populations derived from a single couple reach a stable equilibrium (as far as viability and fertility are concerned) identical to that of the original wild populations. The founder characteristics determined only the way in which the equilibrium is reached. The couple, therefore, possesses all the potentialities necessary to the establishment of a new population. The initial founder and inbreeding effects on fertility and viability characteristics are lost after 5-15 generations in the uniform environment of the laboratory.

These results agree with the predictions of the chromosomal inbreeding theory and raise the problem of inbreeding in small populations and of new area colonization by a small number of individuals. We conclude that consanguinity pressure in limited size populations, environmental influences if effective size becomes great, must be responsible for divergences observed between such populations derived from a single or few couples. The first category of population are hardly or not sensitive to inbreeding at the equilibrium level whereas the second are sensitive to inbreeding when this equilibrium value is reached.
} 


\section{RÉFÉRENCES BIBLIOGRAPHIQUES}

Bifmont C., r972. Effets différents sur la fertilité de trois croisements conduisant au même degré de consanguinité chez Drosophila melanogaster. C. R. Acad. Sci., 275, Série D, ro79-1082.

Bı́monr C., I974a. Étude d'une souche sauvage de Drosophila melanogaster insensible à la consanguinité au niveau des premiers stades du développement embryonnaire. C. R. Acad. Sci., 278, Série D, 32473250.

Bı́́mont C., $1974 b$. Un système d'interactions chromosomiques pour le contrôle du développement embryonnaire. Mech. Age. Dev., 3 (5/6), 29I-299.

Biemont C., Bouffette A. R., Bouffette J., 1974. Théorie chromosomique de l'inbreeding. Modèle probabiliste. Bull. Math. Biol., 36, 4I7-434.

Boesiger E., 1969. Homéostase du développement et homéostase génétique. Année Biol., 8, 58I-6I4.

Bouffette J., Bouffette A. R., r975. Coefficient de dépression et croisements au hasard. Ann. Génét. Sêl. anim. (sous presse).

Crow J. F., Kimura M., r97o. An introduction to population genetics theory. Harper et Row. New York.

David J., I959. Étude quantitative du développement de la Drosophile élevée en milieu axénique. Bull. Biol. Fr. Belg., 98, 472-505.

Dobzhansky T., 1970. Genetics of the evolutionary process. Columbia University Press, New York, London.

Dobzhansky T., Pavlovsky 0 , I957. An experimental study of interaction between genetic drift and natural selection. Evolution, 11, 311-319.

Fischer R. A., I949. The theory of inbreeding. Oliver and Boyd. Edinburg, London.

Haymad B. I., Mather K., I953. The progress of inbreeding when homozygotes are at a disadvantage. Heredity, 7, $165-183$.

Hosgood S. M. W., Parsons P. A., 197r. Genetic heterogeneity among the founders of laboratory populations of Drosophila. IV. Scutellar chaetae in different environments. Genetica, 42, 42-52.

KING H. D., r9I8. Studies on inbreeding. J. Exp. Zool., 26, 3-54.

Lerner J. M., 1954. Genetics homeostasis. Oliver and Boyd. Edinburg.

MAYr E., 1963. Animal species and Evolution. Belknap. Cambridge.

Ney M., Maruyama T., Chakraborty R., 1975. The bottleneck effect and genetic variability in populations. Evolution, 29, I-Io.

Parsons P. A., Hosgood S. M. W., I967. Genetic heterogeneity among the founders of laboratory populations of Drosophila. I. Scutellar chaetae. Genetica, 38, 328-339.

PRAKASH S., 1973. Patterns of gerie variation in central and marginal populations of Drosophila robusta. Genetics, 75, 347-369.

Solima-Simmons A., I966. Experiments on random genetic drift and natural selection in Drosophila pseudoobscura. Evolution, 20, 100. 